\title{
Results on Solutions for Several $q$-Painlevé Difference Equations concerning Rational Solutions, Zeros, and Poles
}

\author{
Bu Sheng Li, ${ }^{1}$ Rui Ying, ${ }^{2}$ Xiu Min Zheng $\mathbb{D}^{3},{ }^{3}$ and Hong Yan Xu $\mathbb{D}^{4}$ \\ ${ }^{1}$ Department of Informatics and Engineering, Jingdezhen Ceramic Institute, Jingdezhen 333403, Jiangxi, China \\ ${ }^{2}$ Basic Department, Shangrao Preschool Education College, Shangrao 334001, Jiangxi, China \\ ${ }^{3}$ Institute of Mathematics and Information Science, Jiangxi Normal University, Nanchan 330022, Jiangxi, China \\ ${ }^{4}$ School of Mathematics and Computer Science, Shangrao Normal University, Shangrao 334001, Jiangxi, China \\ Correspondence should be addressed to Hong Yan Xu; xhyhhh@126.com
}

Received 30 May 2020; Revised 24 July 2020; Accepted 3 August 2020; Published 1 September 2020

Academic Editor: Basil K. Papadopoulos

Copyright $\odot 2020 \mathrm{Bu}$ Sheng Li et al. This is an open access article distributed under the Creative Commons Attribution License, which permits unrestricted use, distribution, and reproduction in any medium, provided the original work is properly cited.

In this article, we discuss the problem about the properties on solutions for several types of $q$-difference equations and obtain some results on the exceptional values of transcendental meromorphic solutions $f(z)$ with zero order, their $q$-differences $\Delta_{q} f(z)=f(q z)-f(z)$, and divided differences $\Delta_{q} f(z) / f(z)$. In addition, we also investigated the condition on the existence of rational solution for a class of $q$-difference equations. Our theorems are some extensions and supplement to those results given by Liu and Zhang and Qi and Yang.

\section{Introduction and Main Results}

All the time, Painlevé equations have attracted much interest due to the reduction of solution equations, which are solvable by inverse scattering transformations, and they often occur in many physical situations: plasma physics, statistical mechanics, and nonlinear waves. The study of Painlevé equations has spanned more than one hundred years (see [1-3]).

Around 2006, Halburd and Korhonen [4, 5] and Ronkainen [6] used Nevanlinna theory to discuss the following equations:

$$
\begin{aligned}
f(z+1)+f(z-1) & =R(z, f) \\
& =\frac{P(z, f)}{Q(z, f)}, \\
f(z+1) f(z-1) & =R(z, f) \\
& =\frac{P(z, f)}{Q(z, f)},
\end{aligned}
$$

where $R(z, f)$ is rational in $f$ and meromorphic in $z$, respectively, and they singled out the following difference equations:

$$
\begin{aligned}
f(z+1)+f(z-1) & =\frac{a z+b}{f(z)}+c, \\
f(z+1)+f(z-1) & =\frac{(a z+b) f(z)+c}{1-f(z)^{2}}, \\
f(z+1) f(z-1) & =\frac{\eta(z) f(z)^{2}-\lambda(z) f(z)+\mu(z)}{(f(z)-1)(f(z)-v(z))}, \\
f(z+1) f(z-1) & =\frac{\eta(z) f(z)^{2}-\lambda(z) f(z)}{f(z)-1}, \\
f(z+1) f(z-1) & =\frac{\eta(z)(f(z)-\lambda(z))}{(f(z)-1)}, \\
f(z+1) f(z-1) & =h(z) f(z)^{m},
\end{aligned}
$$


where $\eta(z), \lambda(z)$, and $v(z)$ satisfy some conditions. In these equations, equation (2) is called as the difference Painlevé I equation, equation (3) is called as the difference Painlevé II equation, and the last four equations are called as the difference Painlevé III equations.

In the last decade or so, there were a lot of papers focusing on the properties of solutions for difference Painlevé I-IV equations (see [7-11]). For example, Chen and Shon [12] in 2010 considered the difference Painlevé I equation (3) and obtained the following theorem.

Theorem 1 (see [12], Theorem 4). Let $a, b, c$ be constants, where $a, b$ are not both equal to zero. Then, the following holds:

(i) If $a \neq 0$, then (3) has no rational solution.

(ii) If $a=0$ and $b \neq 0$, then (3) has a nonzero constant solution $w(z)=A$, where $A$ satisfies $2 A^{2}-c A-$ $b=0$.

The other rational solution $w(z)$ satisfies $w(z)=(P(z) /$ $Q(z))+A$, where $P(z)$ and $Q(z)$ are relatively prime polynomials and satisfy $\operatorname{deg} P<\operatorname{deg} Q$.

In 2013 and 2018, Zhang and Yi [11] and Du et al. [13] studied the difference Painlevé III equations with the constant coefficients and obtained the result as follows.

Theorem 2 (see $[11,13]$ ). If $f$ is a transcendental finite-order meromorphic solution of

$$
f(z+1) f(z-1)(f(z)-1)^{2}=f(z)^{2}-\lambda f(z)+\mu,
$$

where $\lambda$ and $\mu$ are constants, then the following holds:

$$
\begin{aligned}
& \text { (i) } \tau(f)=\sigma(f) \text {. } \\
& \text { (ii) If } \lambda \mu \neq 0 \text {, then } \lambda(f)=\sigma(f) \text {. } \\
& \text { (iii) For any } \eta \in C /\{0\}, \tau(f(z+\eta))=\sigma(f) \text {. } \\
& \text { (iv) } \lambda(1 / \Delta f)=\lambda(1 /(\Delta f / f))=\sigma(f) \text {. }
\end{aligned}
$$

Ramani et al. [14] in 2003 investigated the existence of transcendental solution of equation

$$
\begin{aligned}
(f(z+1)+f(z))(f(z)+f(z-1)) & =R(z, f) \\
& =\frac{P(z, f)}{Q(z, f)},
\end{aligned}
$$

which is called as difference Painlevé IV equations and obtained the result as follows.

Theorem 3 (see [14]). If the second-order difference equation (9) admits a nonrational meromorphic solution of finite order, then $\operatorname{deg}_{z} P \leq 4$ and $\operatorname{deg}_{z} Q \leq 2$.

Of late, many mathematicians paid considerable attention to the value distribution of solutions for complex $q$-difference equations, which are formed by replacing the $q$-difference $f(q z), q \in \mathrm{C} /\{0,1\}$ with $f(z+c)$ of meromorphic function in some expressions concerning complex difference equations, by utilizing the logarithmic derivative lemma on $q$-difference operators given by Barnett et al. [15] in 2007 (see [16-26]). For example, Qi and Yang [27] considered the following equation:

$$
f(q z)+f\left(\frac{z}{q}\right)=\frac{a z+b}{f(z)}+c,
$$

which can be seen as $q$-difference analogues of (2) and obtained the result as follows.

Theorem 4 (see [27], Theorem 1). Let $f(z)$ be a transcendental meromorphic solution with zero order of equation (10) and $a, b, c$ be three constants such that $a, b$ cannot vanish simultaneously. Then, the following holds:

(i) $f(z)$ has infinitely many poles.

(ii) If $a \neq 0$ and any $d \in C$, then $f(z)-d$ has infinitely many zeros.

(iii) If $a=0$ and $f(z)$ takes a finite value A finitely often, then $A$ is a solution of $2 z^{2}-c z-b=0$.

In 2018, Liu and Zhang [28] further investigated the following equation:

$$
Y(\omega z)+Y(z)+Y\left(\frac{z}{\omega}\right)=\frac{\xi z+o}{Y(z)}+v,
$$

and obtained the result as follows.

Theorem 5 (see [28], Theorem 1). Let $Y(z)$ be a transcendental meromorphic solution with zero order of (11) and $\xi, o, \nu$ be three constants such that $\xi, o$ cannot vanish simultaneously. Then, the following holds:

(i) $Y(z)$ has infinitely many poles.

(ii) For any finite value $B$, if $\xi=0$, then $Y(z)-B$ has infinitely many zeros.

(iii) If $\xi=0$ and $Y(z)-A$ has finite zeros, then $A$ is a solution of $3 z^{2}-o-v z=0$.

Motivated by the idea $[27,28]$, a natural question is what is the result if we give $q$-difference analogues of (9). For this question, our main aim of this article is further to investigate some properties of meromorphic solutions for some $q$-Painlevé difference IV equations. It seems that this topic has never been treated before.

In what follows, it should be assumed that the readers are familiar with the fundamental results and the standard notations in the theory of Nevanlinna value distribution (see Hayman [29], Yang [30], and Yi and Yang [31]). Let $f$ be a meromorphic function, and we denote $\sigma(f), \lambda(f)$, and $\lambda(1 / f)$ to be the order, the exponent of convergence of zeros, and the exponent of convergence of poles of $f(z)$, respectively, and denote $\tau(f)$ to be the exponent of convergence of fixed points of $f(z)$, which is defined by

$$
\tau(f)=\lim \sup _{r \longrightarrow+\infty} \frac{\log N(r, 1 /(f(z)-z))}{\log r} .
$$


In addition, we use $S(r, f)$ denotes any quantity satisfying $S(r, f)=o(T(r, f))$ for all $r$ on a set $F$ of logarithmic density 1 , and the logarithmic density of a set $F$ is defined by

$$
\lim \sup _{r \rightarrow \infty} \frac{1}{\log r} \int_{[1, r] \cap F} \frac{1}{t} \mathrm{dt} .
$$

Now, our main results are listed as follows.

Theorem 6. Let $R(z)=A(z) / B(z)$ be an irreducible rational function, and let $q(\neq 0) \in C$ and $|q| \neq 1$, and

$$
\begin{aligned}
{[f(q z)+f(z)]\left[f(z)+f\left(\frac{z}{q}\right)\right] } & =R(z) \\
& =\frac{A(z)}{B(z)},
\end{aligned}
$$

where $A(z), B(z)$ are polynomials with $\operatorname{deg}_{z} A(z)=a$ and $\operatorname{deg}_{z} B(z)=b$.

(i) Suppose that $a \geq b$ and $a-b$ are even numbers or zero. If equation (14) has an irreducible rational solution $f(z)=\mu(z) / \nu(z)$, where $\mu(z), \nu(z)$ are polynomials with $\operatorname{deg}_{z} \mu(z)=\mu$ and $\operatorname{deg}_{z} \nu(z)=\nu$, then the following holds:

$$
\mu-v=\frac{a-b}{2} .
$$

(ii) Suppose that $b \geq a$ and $b-a$ are even numbers or zero. If equation (14) has an irreducible rational solution $f(z)=\mu(z) / \nu(z)$, where $\mu(z), \nu(z)$ are polynomials with $\operatorname{deg}_{z} \mu(z)=\mu$ and $\operatorname{deg}_{z} \nu(z)=\nu$, then

$$
\nu-\mu=\frac{b-a}{2} \text {. }
$$

(iii) If $|a-b|$ is an odd number, then equation (14) has no rational solution.

Theorem 7. For $q(\neq 0) \in C$ and $|q| \neq 1$, let $f(z)$ be a transcendental meromorphic solution with zero order of equation

$$
[f(q z)+f(z)]\left(f(z)+f\left(\frac{z}{q}\right)\right)=a f(z)^{2},
$$

where $a(\neq 0,4)$ is a constant. Let $\Delta_{q} f=f(q z)-f(z)$. Then, the following holds:

(i) Both $f$ and $\Delta_{q} f$ have no nonzero finite Nevanlinna exceptional value.

(ii) If $q+(1 / q) \neq a-2$, then $f(\eta z)$ and $\Delta_{q} f(\eta z)$ have infinitely many fixed points and $\tau(f(\eta z))=\tau\left(\Delta_{q} f(\eta z)\right)=\sigma(f)$ for any nonzero constant $\eta$.

Theorem 8. For $q(\neq 0) \in C$ and $|q| \neq 1$, and let $f(z)$ be a transcendental meromorphic solution with zero order of equation

$$
[f(q z)+f(z)]\left[f(z)+f\left(\frac{z}{q}\right)\right]=d(z) f(z),
$$

where $d(z)$ is a nonconstant rational function satisfying that $d(q z) / d(z)$ is not a constant. Then, the following holds:

(i) Both $f$ and $\Delta_{q} f / f$ have no Nevanlinna exceptional value.

(ii) $\Delta_{q} f$ has infinitely many poles and zeros, and $\lambda\left(1 / \Delta_{q} f\right)=\lambda\left(\Delta_{q} f\right)=\sigma(f)$.

(iii) $\Delta_{q} f / f$ has infinitely many fixed points and $\tau\left(\Delta_{q} f / f\right)=\sigma(f)$.

\section{Proof of Theorem 6}

Proof: assume that (14) has a rational solution $f(z)=\mu(z) / v(z)$ and has poles $z_{1}, z_{2}, \ldots, z_{k}$. Then, $f(z)$ can be represented in the following form:

$$
\begin{aligned}
f(z)=\frac{\mu(z)}{\nu(z)}= & \sum_{j=1}^{k}\left[\frac{c_{j \lambda_{j}}}{\left(z-z_{j}\right)^{\lambda_{j}}}+\cdots+\frac{c_{j 1}}{\left(z-z_{j}\right)}\right] \\
& +a_{0}+a_{1} z+\cdots+a_{s} z^{s},
\end{aligned}
$$

where $c_{j \lambda_{j}}(\neq 0), \ldots, c_{j 1}(j=1,2, \ldots, k)$ and $a_{0}, a_{1}, \ldots, a_{s}$ are constants; $z_{j}(j=1,2, \ldots, k)$ are poles of $f(z)$ with multiplicity $\lambda_{j}$, respectively.

(i) Suppose that $a>b$ and $a-b$ are even numbers. Then, in view of (14) and (19), it yields

$$
\left(\frac{\mu(q z)}{\nu(q z)}+\frac{\mu(z)}{v(z)}\right)\left(\frac{\mu(z)}{v(z)}+\frac{\mu(z / q)}{v(z / q)}\right)=\frac{A(z)}{B(z)} .
$$

If $\operatorname{deg}_{z} \mu(z)=\mu<\nu=\operatorname{deg}_{z} \nu(z)$, then for $z \longrightarrow \infty$, it follows

$$
\begin{gathered}
\frac{\mu(q z)}{\nu(q z)} \longrightarrow 0, \\
\frac{\mu(z)}{\nu(z)} \longrightarrow 0, \\
\frac{\mu(z / q)}{\nu(z / q)} \longrightarrow 0 .
\end{gathered}
$$

However, $A(z) / B(z) \longrightarrow \infty$ as $z \longrightarrow \infty$; thus, from (20), we can get a contradiction easily.

If $\mu=\nu$, then let $z \longrightarrow \infty$, and it leads to

$$
\begin{gathered}
\frac{\mu(q z)}{\nu(q z)} \longrightarrow \alpha, \\
\frac{\mu(z)}{\nu(z)} \longrightarrow \alpha, \\
\frac{\mu(z / q)}{\nu(z / q)} \longrightarrow \alpha,
\end{gathered}
$$


where $\alpha$ is a nonzero constant. Thus, let $z \longrightarrow+\infty$; in view of (20), we also get a contradiction. So, it follows $\mu>v$. Thus, assume that $a_{s} \neq 0,(s \geq 1)$, where $s=\mu-v$. As $z \longrightarrow \infty$, it yields

$$
\begin{gathered}
f(z)=a_{s} z^{s}(1+o(1)), \\
f(q z)=a_{s} q^{s} z^{s}(1+o(1)), \\
f\left(\frac{z}{q}\right)=a_{s} q^{-s} z^{s}(1+o(1)), \\
\frac{A(z)}{B(z)}=\beta z^{a-b}(1+o(1)),
\end{gathered}
$$

where $\beta(\neq 0)$ is a constant, and it follows now in view of (20) that

$$
\left(2+q^{s}+q^{-s}\right) a_{s}^{2} z^{2 s}(1+o(1))=\beta z^{a-b}(1+o(1)),
$$

as $z \longrightarrow \infty$. Since $|q| \neq 1$, then $q^{s}+2+q^{-s} \neq 0$. Hence, it follows from (24) that

$$
\begin{aligned}
\mu-\nu & =s \\
& =\frac{a-b}{2} .
\end{aligned}
$$

Next, assume that $a=b$. As $z \longrightarrow \infty$, it follows

$$
\frac{A(z)}{B(z)}=\beta(1+o(1)) \text {, }
$$

where $\beta(\neq 0)$ is a constant. If $\mu<v$, then by using the same argument as above, we get a contradiction. If $\mu \geq v$, then we assume that $a_{s} \neq 0,(s \geq 1)$. By using the same argument as above, we conclude

$$
\left[q^{s}+2+q^{-s}\right] a_{s}^{2} z^{2 s}=\beta(1+o(1))
$$

as $z \longrightarrow \infty$. Thus, if $\mu>v$, then in view of (27), we can get a contradiction; if $\mu=\nu$, then we have

$$
\begin{aligned}
\mu-\nu & =0 \\
& =\frac{a-b}{2} .
\end{aligned}
$$

(ii) Suppose that $b>a$ and $b-a$ are even numbers. Then, in view of (14) and (19), we get (20).

If $\mu>v$, then for $z \longrightarrow \infty$, it leads to

$$
\begin{aligned}
& \frac{\mu(q z)}{\nu(q z)} \longrightarrow \infty, \\
& \frac{\mu(z)}{\nu(z)} \longrightarrow \infty, \\
& \frac{\mu(z / q)}{\nu(z / q)} \longrightarrow \infty .
\end{aligned}
$$

However, $A(z) / B(z) \longrightarrow 0$ as $z \longrightarrow \infty$; thus, from (20), we can get a contradiction easily.

If $\mu=\nu$, then let $z \longrightarrow \infty$, it follows

$$
\begin{gathered}
\frac{\mu(q z)}{\nu(q z)} \longrightarrow \alpha, \\
\frac{\mu(z)}{\nu(z)} \longrightarrow \alpha, \\
\frac{\mu(z / q)}{\nu(z / q)} \longrightarrow \alpha,
\end{gathered}
$$

where $\alpha$ is a nonzero constant. Thus, let $z \longrightarrow+\infty$; in view of (20), we also get a contradiction. Thus, $\mu<\nu$. We rewrite (14) as the following form:

$$
\begin{aligned}
B(z)\left[\mu(q z) \mu(z) \nu\left(\frac{z}{q}\right) \nu(z)+\mu(q z) \mu\left(\frac{z}{q}\right) \nu(z)^{2}\right. \\
\left.\quad+\mu(z)^{2} \nu(q z) \nu\left(\frac{z}{q}\right)+\mu(z) \mu\left(\frac{z}{q}\right) \nu(q z) \nu(z)\right] \\
=A(z) \nu(z)^{2} v(q z) \nu\left(\frac{z}{q}\right) .
\end{aligned}
$$

Denote

$$
\left\{\begin{array}{l}
A(z)=\xi_{a} z^{a}+\cdots \\
B(z)=\delta_{b} z^{b}+\cdots \\
\mu(z)=\gamma_{\mu} z^{\mu}+\cdots \\
\nu(z)=\zeta_{\nu} z^{\nu}+\cdots
\end{array}\right.
$$

where $a \geq 1, b \geq 0$, and $\mu \geq 0, \nu \geq 1$ are all nonnegative integers. Thus, in view of (31) and (32), we can deduce

$$
\left(q^{\mu-\nu}+2+q^{\nu-\mu}\right) \delta_{b} \gamma_{\mu}^{2} \zeta_{\nu}^{2} z^{2(\mu+\nu)+b}+\cdots=\xi_{a} \zeta_{\nu}^{4} z^{4 \nu+a} .
$$

Since $|q| \neq 1$, then $q^{\mu-\nu}+2+q^{\nu-\mu} \neq 0$. Thus, by combining with this and (33), we have

$$
2(\mu+v)+b=4 v+a, \quad \text { that is } v-\mu=\frac{b-a}{2},
$$

and $\zeta_{\nu}^{2} / \gamma_{\mu}^{2}=\left(\delta_{b} / \xi_{a}\right)\left(q^{\mu-\nu}+2+q^{\nu-\mu}\right)$.

(iii) If $a>b$, then $|a-b|=a-b$ is an odd number. Assume that $f(z)=\mu(z) / \nu(z)$ is a rational solution of (14). In view of the conclusion of Theorem 6 (i), it follows $\mu-v=(a-b) / 2$. This means a contradiction with the assumption that $a-b$ is an odd number. Thus, (14) has no rational solution.

If $a<b$, then $|a-b|=b-a$ is an odd number. Similar to the above argument, we also conclude that (14) has no rational solution. 
Therefore, this completes the proof of Theorem 6 .

\section{Proof of Theorem 7}

We first introduce some notations and some basic results about Nevanlinna theory, which can be used in Section 3 and Section 4. Let $f$ be a meromorphic function in $\mathrm{C}$, the Nevanlinna characteristic $T(r, f)$, which encodes information about the distribution of values of $f$ on the disk $|z| \leq r$, is defined by

$$
T(r, f)=m(r, f)+N(r, f) .
$$

The proximity function $m(r, f)$ is defined by

$$
\begin{aligned}
m(r, f) & =m(r, \infty, f) \\
& =\frac{1}{2 \pi} \int_{0}^{2 \pi} \log ^{+}\left|f\left(\mathrm{re}^{i \theta}\right)\right| \mathrm{d} \theta,
\end{aligned}
$$

where $\log ^{+} x=\max \{0, \log x\}$ and

$$
\begin{aligned}
N(r, f) & =N(r, \infty, f) \\
& =\int_{0}^{r} \frac{n(t, f)-n(0, f)}{t} \mathrm{~d} t+n(0, f) \log r,
\end{aligned}
$$

where $n(r, f)$ is the number of poles of $f$ in the circle $|z|=r$, counted according to multiplicities.

Let $a \in C \cup\{\infty\}$, and the deficiency of $a$ with respect to $f(z)$ is defined by

$$
\begin{aligned}
\delta(a, f) & =\liminf _{r \longrightarrow+\infty} \frac{m(r,(1 / f-a))}{T(r, f)} \\
& =1-\limsup _{r \longrightarrow+\infty} \frac{N(r,(1 / f-a))}{T(r, f)} .
\end{aligned}
$$

If $\delta(a, f)>0$, then the complex number $a$ is called the Nevanlinna exceptional value. And the order $\sigma(f)$, the exponent of convergence of zeros $\lambda(f)$, and the exponent of convergence of poles $\lambda(1 / f)$ of $f(z)$ are defined by

$$
\begin{aligned}
\sigma(f) & =\limsup _{r \longrightarrow+\infty} \frac{\log ^{+} T(r, f)}{\log r}, \\
\lambda(f) & =\limsup _{r \longrightarrow+\infty} \frac{\log ^{+} N(r, 1 / f)}{\log r}, \\
\lambda\left(\frac{1}{f}\right) & =\limsup _{r \longrightarrow+\infty} \frac{\log ^{+} N(r, f)}{\log r} .
\end{aligned}
$$

Besides, we also use some properties of $T(r, f), m(r, f)$, and $N(r, f)$ such as

$$
\begin{aligned}
T\left(r, \frac{1}{f-a}\right) & =T(r, f)+O(1), \\
m\left(r, \prod_{j=1}^{p} f_{j}\right) & \leq \sum_{j=1}^{p} m\left(r, f_{j}\right), m\left(r, \sum_{j=1}^{p} f_{j}\right) \\
& \leq \sum_{j=1}^{p} N\left(r, f_{j}\right)+\log p, \\
N\left(r, \prod_{j=1}^{p} f_{j}\right) & \leq \sum_{j=1}^{p} N\left(r, f_{j}\right), N\left(r, \sum_{j=1}^{p} f_{j}\right) \leq \sum_{j=1}^{p} N\left(r, f_{j}\right), \\
T\left(r, \prod_{j=1}^{p} f_{j}\right) & \leq \sum_{j=1}^{p} T\left(r, f_{j}\right), T\left(r, \sum_{j=1}^{p} f_{j}\right) \\
& \leq \sum_{j=1}^{p} T\left(r, f_{j}\right)+\log p,
\end{aligned}
$$

where $f_{j}(z)(j=1,2, \ldots, p)$ are $p$ meromorphic functions and $a \in \mathrm{C}$ and require some lemmas as follows.

Lemma 1 (see [15], Theorem 2). Let $f$ be a nonconstant zero-order meromorphic solution of $P(z, f)=0$, where $P(z, f)$ is a q-difference polynomial in $f(z)$. If $P(z, a) \neq 0$ for slowly moving target $a(z)$, then

$$
m\left(r, \frac{1}{f-a}\right)=S(r, f),
$$

where $S(r, f)$ denotes any quantity satisfying $S(r, f)=o(T(r, f))$ for all $r$ on a set $F$ of logarithmic density 1.

Remark 1. For $q \in \mathrm{C} /\{0,1\}$, a polynomial in $f(z)$ and finitely many of its $q$-shifts $f(q z), \ldots, f\left(q^{n} z\right)$ with meromorphic coefficients in the sense that their Nevanlinna characteristic functions are $o(T(r, f))$ on a set $F$ of logarithmic density 1 and can be called as a $q$-difference polynomial of $f$.

Lemma 2 (see [24], Theorems 1 and 3). Let $f(z)$ be a nonconstant zero-order meromorphic function and $q \in C /\{0\}$. Then,

$$
\begin{aligned}
& T(r, f(q z))=(1+o(1)) T(r, f(z)), \\
& N(r, f(q z))=(1+o(1)) N(r, f(z)),
\end{aligned}
$$

on a set of lower logarithmic density 1. 
The proof of Theorem 7: (i) suppose that $f(z)$ is a transcendental meromorphic solution of equation (17), then in view of (17), let

$$
P_{1}(z, f):=[f(q z)+f(z)]\left[f(z)+f\left(\frac{z}{q}\right)\right]-a f(z)^{2} \equiv 0 .
$$

For any given constant $d \in \mathrm{C} /\{0\}$ and with a view of $a \neq 4$, it follows

$$
P_{1}(z, d)=4 d^{2}-a d^{2} \neq 0 .
$$

In view of $P_{1}(z, d) \not \equiv 0$ and by Lemma 1 , we conclude that $m(r,(1 / f-d))=S(r, f)$. This leads to

$$
N\left(r, \frac{1}{f-d}\right)=T(r, f)+S(r, f),
$$

which implies $\delta(d, f)=0$. Thus, $f(z)$ has no nonzero finite Nevanlinna exceptional value.

Since $f(z)$ is of zero order and $\Delta_{q} f=f(q z)-f(z)$, then by Lemma 2, it follows $T\left(r, \Delta_{q} f\right) \leq 2 T(r, f)+S(r, f)$, which means that $\Delta_{q} f$ if of zero order. In view of (17), it follows

$$
\left[f\left(q^{2} z\right)+f(q z)\right][f(q z)+f(z)]=a f(q z)^{2} .
$$

With (17) subtraction, it leads to

$$
\begin{aligned}
& {[f(q z)+f(z)]\left[f\left(q^{2} z\right)+f(q z)-f(z)-f\left(\frac{z}{q}\right)\right]} \\
& \quad=a[f(q z)+f(z)][f(q z)-f(z)] .
\end{aligned}
$$

From (17), we see that $f(q z)+f(z) \equiv 0$. Otherwise, it leads to $f(z) \equiv 0$, a contradiction. Thus, the above equality means

$$
f\left(q^{2} z\right)+f(q z)-f(z)-f\left(\frac{z}{q}\right)=a[f(q z)-f(z)],
$$

that is,

$$
\Delta_{q} f(q z)+(2-a) \Delta_{q} f(z)+\Delta_{q} f\left(\frac{z}{q}\right)=0 .
$$

Denote

$$
\begin{aligned}
P_{2}\left(z, \Delta_{q} f\right):= & \Delta_{q} f(q z)+(2-a) \Delta_{q} f(z) \\
& +\Delta_{q} f\left(\frac{z}{q}\right) \equiv 0 .
\end{aligned}
$$

For any given constant $d \in \mathrm{C} /\{0\}$, then from (50), we have $P_{2}(z, d)=(4-a) d$. Hence, with a view of $a \neq 4$, it follows $P_{2}(z, d) \equiv 0$. Thus, by Lemma 1 , we have $m\left(r, 1 / \Delta_{q} f-d\right)=S\left(r, \Delta_{q} f\right)$, and this leads to

$$
N\left(r, \frac{1}{\Delta_{q} f-d}\right)=T\left(r, \Delta_{q} f\right)+S\left(r, \Delta_{q} f\right)
$$

which implies that $\delta\left(d, \Delta_{q} f\right)=0$. Thus, $\Delta_{q} f$ has no nonzero finite Nevanlinna exceptional value:

(ii) Replacing $z$ by $\eta z$ in (17), we have

$$
[f(q \eta z)+f(\eta z)]\left[f(\eta z)+f\left(\frac{\eta z}{q}\right)\right]=a f(\eta z)^{2} .
$$

Let $g_{1}(z)=f(\eta z)$, it yields

$$
\left[g_{1}(q z)+g_{1}(z)\right]\left[g_{1}(z)+g_{1}\left(\frac{z}{q}\right)\right]=a g_{1}(z)^{2} \text {. }
$$

Set

$$
\begin{aligned}
P_{3}\left(z, g_{1}\right):= & {\left[g_{1}(q z)+g_{1}(z)\right] } \\
& \cdot\left[g_{1}(z)+g_{1}\left(\frac{z}{q}\right)\right]-a g_{1}(z)^{2} \equiv 0 .
\end{aligned}
$$

Thus, it follows $P_{3}(z, z)=\left(\left((q+1)^{2} / q\right)-a\right) z^{2}$, and with a view of $q+(1 / q) \neq a-2$, we have $P_{3}(z, z) \equiv 0$. By applying Lemma 1, it yields $m\left(r, 1 /\left(g_{1}(z)-z\right)\right)=S\left(r, g_{1}\right)$. Thus, in view of Lemma 2 , this leads to

$$
\begin{aligned}
N\left(r, \frac{1}{f(\eta z)-z}\right) & =N\left(r, \frac{1}{g_{1}(z)-z}\right) \\
& =T\left(r, g_{1}(z)\right)+S\left(r, g_{1}\right) \\
& =T(r, f(\eta z))+S(r, f(\eta z)) \\
& =T(r, f)+S(r, f),
\end{aligned}
$$

which implies that $f(\eta z)$ has infinitely many fixed points and $\tau(f(\eta z))=\sigma(f)$.

In view of (48), set $g_{2}(z)=\Delta_{q} f(\eta z)$ and

$$
P_{4}\left(z, g_{2}\right):=g_{2}(q z)+(2-a) g_{2}(z)+g_{2}\left(\frac{z}{q}\right) \equiv 0,
$$

then $\quad P_{4}(z, z)=[q+(2-a)+(1 / q)] z$. Since $q+(1 / q) \neq a-2$, then $P_{4}(z, z) \equiv 0$. By applying Lemma 1 , we have $m\left(r,\left(1 /\left(g_{2}(z)-z\right)\right)\right)=S\left(r, g_{2}\right)$. Thus, in view of Lemma 2 , this leads to

$$
\begin{aligned}
N\left(r, \frac{1}{\Delta_{q} f(\eta z)-z}\right) & =N\left(r, \frac{1}{g_{2}(z)-z}\right) \\
& =T\left(r, g_{2}(z)\right)+S\left(r, g_{1}\right) \\
& =T\left(r, \Delta_{q} f(\eta z)\right)+S(r, f(\eta z)),
\end{aligned}
$$

which implies that $\Delta_{q} f(\eta z)$ has infinitely many fixed points and $\tau\left(\Delta_{q} f(\eta z)\right)=\sigma(f)$.

Therefore, the proof of Theorem 7 is completed. 


\section{Proof of Theorem 8}

Lemma 3 (see [15], Theorem 1). Let $f(z)$ be a nonconstant zero-order meromorphic function and $q \in C /\{0\}$. Then,

$$
m\left(r, \frac{f(q z)}{f(z)}\right)=S(r, f) .
$$

Lemma 4 (see [17], Theorem 2.5). Let $f$ be a transcendental meromorphic solution of order zero of a q-difference equation of the form

$$
U_{q}(z, f) P_{q}(z, f)=Q_{q}(z, f),
$$

where $U_{q}(z, f), P_{q}(z, f)$, and $Q_{q}(z, f)$ are $q$-difference polynomials such that the total degree $\operatorname{deg} U_{q}(z, f)=n$ in $f(z)$ and its $q$-shifts, whereas $\operatorname{deg} Q_{q}(z, f) \leq n$. Moreover, we assume that $U_{q}(z, f)$ contains just one term of maximal total degree in $f(z)$ and its q-shifts. Then,

$$
m\left(r, P_{q}(z, f)\right)=S(r, f) .
$$

Proof of Theorem 8:. (i) suppose that $f(z)$ is a transcendental meromorphic solution of equation (18). We firstly prove that $\Delta_{q} f / f$ has no Nevanlinna exceptional value. Equation (18) can be rewritten as

$$
\left(\frac{f(q z)}{f(z)}+1\right)\left(\frac{f(z / q)}{f(z)}+1\right)=\frac{d(z)}{f(z)} .
$$
follows

Set $g_{3}(z)=f(q z) / f(z)$. In view of (61) and Lemma 2, it

$$
\begin{aligned}
T(r, f(z))= & T\left(r, \frac{d(z)}{f(z)}\right)+O(\log r) \leq T\left(r, \frac{f(q z)}{f(z)}\right) \\
& +T\left(r, \frac{f(z / q)}{f(z)}\right)+O(\log r) \\
= & T\left(r, g_{3}(z)\right)+T\left(r, g_{3}\left(\frac{z}{q}\right)\right)+O(\log r) \\
= & 2 T\left(r, g_{3}\right)+S\left(r, g_{3}\right) \\
= & 2 T\left(r, \frac{\Delta_{q} f}{f}\right)+S(r, f) .
\end{aligned}
$$

And with a view of $T\left(r, \Delta_{q} f / f\right) \leq 2 T(r, f)+S(r, f)$, we thus conclude that $\Delta_{q} f / f$ is transcendental and of order zero, and $d(z)$ is small with respect to $g_{3}(z)$.

Replacing $z$ by $q z$ in (18), it follows

$$
\left[f\left(q^{2} z\right)+f(q z)\right][f(q z)+f(z)]=d(q z) f(q z) .
$$

By combining with (18), we have

$$
\frac{f\left(q^{2} z\right)+f(q z)}{f(z)+f(z / q)}=\frac{d(q z) f(q z)}{d(z) f(z)} .
$$

Since $g_{3}(z)=f(q z) / f(z)$, then it yields

$$
\begin{aligned}
f(q z) & =g_{3}(z) f(z), \\
f\left(q^{2} z\right) & =g_{3}(q z) f(q z) \\
& =g_{3}(z) g_{3}(q z) f(z), \\
f\left(\frac{z}{q}\right) & =\frac{f(z)}{g_{3}(z / q)} .
\end{aligned}
$$

Substituting (65) into (64), we obtain

$$
\frac{g_{3}(z) g_{3}(q z) f(z)+g_{3}(z) f(z)}{f(z)+\left(f(z) / g_{3}(z / q)\right)}=\frac{d(q z)}{d(z)} g_{3}(z) \text {, }
$$

that is,

$$
g_{3}\left(\frac{z}{q}\right)\left(g_{3}(q z)+1\right)=\frac{d(q z)}{d(z)}\left(g\left(\frac{z}{q}\right)+1\right) .
$$

By applying Lemma 4 for (67), it follows $m\left(r, g_{3}(z / q)\right)=S\left(r, g_{3}\right)$. This leads to

$$
N\left(r, g_{3}\left(\frac{z}{q}\right)\right)=T\left(r, g_{3}\right)+S\left(r, g_{3}\right) \text {. }
$$

Thus, in view of Lemma 2, it yields

$$
\begin{aligned}
(1+o(1)) N\left(r, g_{3}\right) & =N\left(r, g_{3}\left(\frac{z}{q}\right)\right) \\
& =T\left(r, g_{3}\left(\frac{z}{q}\right)\right)+S\left(r, g_{3}\right) \\
& =(1+o(1)) T\left(r, g_{3}\right)+S\left(r, g_{3}\right) .
\end{aligned}
$$

This shows

$$
\begin{aligned}
N\left(r, \frac{\Delta_{q} f}{f}\right) & =N\left(r, g_{3}\right) \\
& =T\left(r, g_{3}\right)+S\left(r, g_{3}\right) \\
& =T\left(r, \frac{\Delta_{q} f}{f}\right)+S\left(r, \frac{\Delta_{q} f}{f}\right),
\end{aligned}
$$

which implies $\delta\left(\infty, \Delta_{q} f / f\right)=0$.

Set

$$
\begin{aligned}
P_{5}\left(z, g_{3}\right):= & g_{3}\left(\frac{z}{q}\right)\left(g_{3}(q z)+1\right) \\
& -\frac{d(q z)}{d(z)}\left(g\left(\frac{z}{q}\right)+1\right) \equiv 0 .
\end{aligned}
$$

For any constant $\varrho \in C /\{-2\}$, we have 


$$
\begin{aligned}
P_{5}(z, \varrho+1) & =(\varrho+1)(\varrho+1+1)-\frac{d(q z)}{d(z)}(\varrho+1+1) \\
& =(\varrho+2)\left(\varrho+1-\frac{d(q z)}{d(z)}\right) .
\end{aligned}
$$

Since $d(q z) / d(z)$ is not a constant, then $P_{5}(z, \varrho+1) \not \equiv$. By Lemma 1, it follows

$$
\begin{aligned}
m\left(r, \frac{1}{\left(\Delta_{q} f / f\right)-\varrho}\right) & =m\left(r, \frac{1}{g_{3}-\varrho-1}\right) \\
& =S\left(r, g_{3}\right)=S\left(r, \frac{\Delta_{q} f}{f}\right) .
\end{aligned}
$$

This means

$$
N\left(r, \frac{1}{\left(\Delta_{q} f / f\right)-\varrho}\right)=T\left(r, \frac{\Delta_{q} f}{f}\right)+S\left(r, \frac{\Delta_{q} f}{f}\right),
$$

which implies that $\delta\left(\varrho, \Delta_{q} f / f\right)=0$ for any constant $\varrho \in C /\{-2\}$.

In view of (18) and Lemma 3, we have

$$
\begin{aligned}
m\left(r, \frac{1}{f(q z)+f(z)}\right) & =m\left(r, \frac{f(z)+f(z / q)}{d(z) f(z)}\right) \\
\leq & m\left(r, \frac{1}{d(z)}\right)+m\left(r, \frac{f(z)+f(z / q)}{f(z)}\right) \\
& +O(1) \\
\leq & S(r, f) .
\end{aligned}
$$

Thus, we can conclude from (18), (75), and Lemma 2 that

$$
\begin{aligned}
N\left(r, \frac{1}{f(q z)+f(z)}\right) & =T\left(r, \frac{1}{f(q z)+f(z)}\right)+S(r, f) \\
& =T\left(r, \frac{1}{d(z)} \frac{f(z)+f(z / q)}{f(z)}\right)+S(r, f) \\
& =T\left(r, \frac{f(z / q)}{f(z)}\right)+O(\log r)+S(r, f) \\
& =T\left(r, \frac{f(q z)}{f(z)}\right)+S(r, f) \\
& =T\left(r, \frac{\Delta_{q} f}{f}\right)+S(r, f) .
\end{aligned}
$$

On the contrary, we can see that the zero of $\left(\Delta_{q} f / f\right)+2$ is the zero of $f(q z)+f(z)$, and the zero of $f(q z)+f(z)$ is also the zero of $\left(\Delta_{q} f / f\right)+2$. Indeed, if $z_{0}$ is a zero of $\left(\Delta_{q} f / f\right)+2$, that is, $\left(\left(f\left(q z_{0}\right)-f\left(z_{0}\right)\right) / f\left(z_{0}\right)\right)+2=0$, then it follows $f\left(q z_{0}\right)+f\left(z_{0}\right)=0$, and this shows that $z_{0}$ is a zero of $f(q z)+f(z)$; if $z_{1}$ is a zero of $f(q z)+f(z)$, that is, $f\left(q z_{1}\right)+f\left(z_{1}\right)=0$, then it follows $\left(\Delta_{q} f\left(q z_{1}\right) / f\left(z_{1}\right)\right)+$ $2=0$, and this shows that $z_{1}$ is a zero of $\left(\Delta_{q} f / f\right)+2$. Thus, by combining with (76), we conclude

$$
\begin{aligned}
N\left(r, \frac{1}{\left(\Delta_{q} f / f\right)+2}\right) & =N\left(r, \frac{1}{f(q z)+f(z)}\right) \\
& =T\left(r, \frac{\Delta_{q} f}{f}\right)+S\left(r, \frac{\Delta_{q} f}{f}\right) .
\end{aligned}
$$

This shows that $\delta\left(-2, \Delta_{q} f / f\right)=0$; thus, by combining with $\delta\left(\infty, \Delta_{q} f / f\right)=0$ and $\delta\left(\varrho, \Delta_{q} f / f\right)=0$ for any $\varrho \in \mathrm{C} /\{-2\}$, we have $\delta\left(\varrho, \Delta_{q} f / f\right)=0$ for any $\varrho \in \mathrm{C}$. So, $\Delta_{q} f / f$ has no Nevanlinna exceptional value.

Next, we prove that $f(z)$ has no Nevanlinna exceptional value.

Firstly, in view of (61) and Lemma 3, we have

$$
\begin{aligned}
m\left(r, \frac{1}{f}\right) & \leq m\left(r, \frac{1}{d(z)}\right)+m\left(r, \frac{f(q z)}{f(z)}\right)+m\left(r, \frac{f(z / q)}{f(z)}\right) \\
& =S(r, f),
\end{aligned}
$$

which implies

$$
N\left(r, \frac{1}{f}\right)=T(r, f)+S(r, f) .
$$

This means $\delta(0, f)=0$.

Secondly, in view of (18), we denote

$$
P_{6}(z, f):=[f(q z)+f(z)]\left[f(z)+f\left(\frac{z}{q}\right)\right]-d(z) f(z) \equiv 0 .
$$

Since $d(z)$ is a nonconstant function, then for any constant $\varrho \in C /\{0\}$, it yields

$$
\begin{aligned}
P_{6}(z, \varrho) & =4 \varrho^{2}-\varrho d(z) \\
& =\varrho(4 \varrho-d(z)) \neq \equiv .
\end{aligned}
$$

Thus, from Lemma 1 , we conclude $m(r, 1 / f-\varrho)=$ $S(r, f)$, which implies

$$
N\left(r, \frac{1}{f-\varrho}\right)=T(r, f)+S(r, f) .
$$

This means that $\delta(\varrho, f)=0$ for any constant $\varrho \in C /\{0\}$. Finally, in view of (77), we have

$$
\begin{aligned}
m\left(r, \frac{1}{\left(\Delta_{q} f / f\right)+2}\right) & =S\left(r, \frac{\Delta_{q} f}{f}\right) \\
& =S(r, f) .
\end{aligned}
$$

Thus, from (18) and (83), it yields 


$$
\begin{aligned}
m\left(r, f(z)+f\left(\frac{z}{q}\right)\right) & =m\left(r, \frac{d(z) f(z)}{f(q z)+f(z)}\right) \\
& =m\left(r, \frac{1}{(f(q z) / f(z))+1}\right)+O(\log r) \\
& =m\left(r, \frac{1}{\left(\Delta_{q} f / f\right)+2}\right)+O(\log r) \\
& =S(r, f) .
\end{aligned}
$$

Hence, by the above equality and in view of (18) and Lemma 3, it leads to

$$
\begin{aligned}
m(r, f) \leq & m\left(r, f(z)+f\left(\frac{z}{q}\right)\right)+m(r, f(q z)+f(z)) \\
& +O(\log r) \\
\leq & 2 m\left(r, f(z)+f\left(\frac{z}{q}\right)\right)+m\left(r, \frac{f(q z)+f(z)}{f(z)+f(z / q)}\right) \\
& +O(\log r) \\
= & S(r, f),
\end{aligned}
$$

which implies

$$
N(r, f)=T(r, f)+S(r, f) .
$$

Hence, $\delta(\infty, f)=0$. Together with $\delta(0, f)=0$ and $\delta(\varrho, f)=0$ for any $\varrho \in C /\{0\}$, we obtain that $f(z)$ has no Nevanlinna exceptional value.

(ii) Since $\quad f(q z)=\Delta_{q} f(z)+f(z) \quad$ and $f(z / q)=f(z)-\Delta_{q} f(z / q)$, then by substituting these into (18), it follows

$$
\left[2 f(z)+\Delta_{q} f(z)\right]\left[2 f(z)-\Delta_{q} f\left(\frac{z}{q}\right)\right]=d(z) f(z) .
$$

If $z_{0}$ is a zero of $f(z)$ and is not a pole of $d(z)$, then in view of (87), we conclude that $z_{0}$ is a zero of $\Delta_{q} f(z)$ or a zero of $\Delta_{q} f(z / q)$. Thus, it follows from Lemma 2 that

$$
\begin{aligned}
N\left(r, \frac{1}{f}\right) & \leq N\left(r, \frac{1}{\Delta_{q} f(z)}\right)+N\left(r, \frac{1}{\Delta_{q} f(z / q)}\right)+N(r, d) \\
& =2 N\left(r, \frac{1}{\Delta_{q} f}\right)+S(r, f) .
\end{aligned}
$$

Thus, by combining with (79), it yields that $\Delta_{q} f(z)$ has infinitely many zeros and $\lambda\left(\Delta_{q} f\right)=\sigma(f)$.
In view of (85) and Lemma 3, we can deduce

$$
\begin{aligned}
m\left(r, \Delta_{q} f\right) & \leq m(r, f)+m\left(r, \frac{\Delta_{q} f}{f}\right)=S(r, f) \\
& =S\left(r, \Delta_{q} f\right),
\end{aligned}
$$

which implies

$$
\begin{aligned}
N\left(r, \Delta_{q} f\right) & =T\left(r, \Delta_{q} f\right)+S\left(r, \Delta_{q} f\right) \\
& =T\left(r, \frac{1}{\Delta_{q} f}\right)+S\left(r, \Delta_{q} f\right) .
\end{aligned}
$$

Thus, by combining with (88) and (79), we have that $\Delta_{q} f(z)$ has infinitely many poles and $\lambda\left(1 / \Delta_{q} f\right)=\sigma(f)$. This proves the conclusions of Theorem 8 (ii).

(iii) In view of (71), it follows

$$
\begin{aligned}
P_{5}(z, z+1) & =\left(\frac{z}{q}+1\right)(q z+1+1)-\frac{d(q z)}{d(z)}\left(\frac{z}{q}+1+1\right) \\
& =\frac{(z+q)(q z+2)}{q}-\frac{d(q z)}{d(z)} \frac{z+2 q}{q} .
\end{aligned}
$$

Since $d(z)$ is a nonconstant rational function, then let $z \longrightarrow \infty$, and we have $d(q z) / d(z) \longrightarrow q^{\kappa}$, where $\kappa=\operatorname{deg}_{z} d$. But, for $\quad q \neq 0, \quad$ it follows $((z+q)(q z+2) / z+2 q) \longrightarrow \infty$ as $z \longrightarrow \infty$. Thus, we can deduce $P_{5}(z, z+1) \equiv 0$. By Lemma 1 , we conclude that

$$
\begin{aligned}
m\left(r \frac{1}{\left(\Delta_{q} f / f\right)-z}\right) & =m\left(r, \frac{1}{g_{3}(z)-1-z}\right) \\
& =S\left(r, g_{3}\right) \\
& =S\left(r, \frac{\Delta_{q} f}{f}\right) .
\end{aligned}
$$

This leads to

$$
N\left(r \frac{1}{\left(\Delta_{q} f / f\right)-z}\right)=T\left(r, \frac{\Delta_{q} f}{f}\right)+S\left(r, \frac{\Delta_{q} f}{f}\right),
$$

which implies that $\Delta_{q} f / f$ has infinitely many fixed points and $\tau\left(\Delta_{q} f / f\right)=\sigma(f)$.

Therefore, this completes the proof of Theorem 8 .

\section{Data Availability}

No data were used to support this study.

\section{Conflicts of Interest}

The authors declare that they have no conflicts of interest. 


\section{Authors' Contributions}

H. Y. Xu and X. M. Zheng conceptualized the study. H. Y. Xu and R. Ying wrote the original draft. H. Y. Xu, B. S. Li, and X. M. Zheng reviewed and edited the manuscript. H. Y. Xu and B. S. Li obtained funding acquisition.

\section{Acknowledgments}

This work was supported by the National Natural Science Foundation of China (no. 11561033), Natural Science Foundation of Jiangxi Province in China (nos. 20181BAB201001 and 20151BAB201008), and Foundation of Education Department of Jiangxi (nos. GJJ190876, GJJ190895, and GJJ191042) of China.

\section{References}

[1] A. S. Fokas, B. Grammaticos, and A. Ramani, "From continuous to discrete Painlevé equations," Journal of Mathematical Analysis and Applications, vol. 180, no. 2, pp. 342-360, 1993.

[2] B. Grammaticos, F. W. Nijhoff, and A. Ramani, "Discrete Painlevé equations," The Painlevé Property, CRM Series in Mathematical Physics, Springer, New York, NY, USA, pp. 413-516, 1999.

[3] P. Painlevé, "Mémoire sur les équations différentielles dont l'intégrale générale est uniforme," Bulletin de la Société mathématique de France, vol. 28, pp. 201-261, 1900.

[4] R. G. Halburd and R. J. Korhonen, "Difference analogue of the lemma on the logarithmic derivative with applications to difference equations," Journal of Mathematical Analysis and Applications, vol. 314, no. 2, pp. 477-487, 2006.

[5] R. G. Halburd and R. J. Korhonen, "Finite-order meromorphic solutions and the discrete Painlevé equations," Proceedings of the London Mathematical Society, vol. 94, no. 2, pp. 443-474, 2007.

[6] O. Ronkainen, "Meromorphic solutions of difference Painlevé equations," Ph.D. thesis, Department of Physics and Mathematics, University of Eastern Finland, Finland, 2010.

[7] Z. X. Chen, "Complex differences and difference equations," Mathematics Monograph Series, p. 29, Science Press, Beijing, China, 2014.

[8] Z.-T. Wen, "Finite order solutions of difference equations, and difference Painlevé equations IV," Proceedings of the American Mathematical Society, vol. 144, no. 10, pp. 4247-4260, 2016.

[9] H. Y. Xu and J. Tu, "Existence of rational solutions for $q$ difference Painlevé equations," Electronic Journal of Differential Equations, vol. 2020, no. 14, pp. 1-14, 2020.

[10] J. Zhang, "Some results on difference Painlevé IV equations," Journal of Difference Equations and Applications, vol. 22, no. 12, pp. 1912-1929, 2016.

[11] J. Zhang and H. Yi, "Properties of meromorphic solutions of Painlevé III difference equations," Advances in Difference Equations, vol. 2013, no. 1, p. 256, 2013.

[12] Z.-X. Chen and K. H. Shon, "Value distribution of meromorphic solutions of certain difference Painlevé equations," Journal of Mathematical Analysis and Applications, vol. 364, no. 2, pp. 556-566, 2010.

[13] Y. F. Du, M. F. Chen, Z. S. Gao, and M. Zhao, "Value distribution of meromorphic solutions of certain difference Painlevé III equations," Advances in Difference Equations, vol. 2018, p. 171, 2018.
[14] A. Ramani, B. Grammaticos, T. Tamizhmani, and K. M. Tamizhmani, "The road to the discrete analogue of the Painlevé property: Nevanlinna meets singularity confinement," Computers \& Mathematics with Applications, vol. 45, no. 6-9, pp. 1001-1012, 2003.

[15] D. C. Barnett, R. G. Halburd, R. J. Korhonen, and W. Morgan, "Nevanlinna theory for the \$q\$-difference operator and meromorphic solutions of \$q $\$$-difference equations," Proceedings of the Royal Society of Edinburgh: Section A Mathematics, vol. 137, no. 3, pp. 457-474, 2007.

[16] G. G. Gundersen, J. Heittokangas, I. Laine, J. Rieppo, and D. Yang, "Meromorphic solutions of generalized Schröder equations," Aequationes Mathematicae, vol. 63, no. 1-2, pp. 110-135, 2002.

[17] I. Laine and C.-C. Yang, "Clunie theorems for difference and q-difference polynomials," Journal of the London Mathematical Society, vol. 76, no. 3, pp. 556-566, 2007.

[18] K. Liu, "Entire solutions of Fermat type $q$-difference differential equations," Electronic Journal of Differential Equations, vol. 2013, no. 59, pp. 1-10, 2013.

[19] M. Ru, "The recent progress in Nevanlinna theory," Journal of Jiangxi Normal University. Natural Sciences Edition, vol. 42, pp. 1-11, 2018.

[20] H. Y. Xu, S. Y. Liu, and Q. P. Li, "Entire solutions for several systems of nonlinear difference and partial differential-difference equations of Fermat-type," Journal of Mathematical Analysis and Applications, vol. 483, no. 2, Article ID 123641, 2020.

[21] H. Y. Xu, S. Y. Liu, and Q. P. Li, “The existence and growth of solutions for several systems of complex nonlinear difference equations," Mediterranean Journal of Mathematics, vol. 16, no. 1, p. 8, 2019.

[22] H. Y. Xu, S. Y. Liu, and X. M. Zheng, "Some properties of meromorphic solutions for $q$-difference equations," Electronic Journal of Differential Equations, vol. 2017, no. 175, pp. 1-12, 2017.

[23] Q. Y. Yuan, J. R. Long, and D. Z. Qin, "The growth of solutions of two certain types of $q$-difference differential equations," Journal of Jiangxi Normal University. Natural Sciences Edition, vol. 44, pp. 6-11, 2020.

[24] J. Zhang and R. Korhonen, "On the Nevanlinna characteristic of $f(q z)$ and its applications," Journal of Mathematical Analysis and Applications, vol. 369, no. 2, pp. 537-544, 2010.

[25] X. M. Zheng and Z. X. Chen, "On properties of $q$-difference equations," Acta Mathematica Scientia, vol. 32, no. 2, pp. 724-734, 2012.

[26] X.-M. Zheng and Z.-X. Chen, "Some properties of meromorphic solutions of $q$-difference equations," Journal of Mathematical Analysis and Applications, vol. 361, no. 2, pp. 472-480, 2010.

[27] X. G. Qi and L. Z. Yang, "Properties of meromorphic solutions of q-difference equations," Electronic Journal of Differential Equations, vol. 2015, no. 59, pp. 1-9, 2015.

[28] Y. Liu and Y. Zhang, "Some results of $₫$-Painlevé difference equation," Advances in Difference Equations, vol. 2018, no. 1, p. $282,2018$.

[29] W. K. Hayman, Meromorphic Functions, Clarendon Press, Oxford, UK, 1964.

[30] L. Yang, Value Distribution Theory, Springer, Berlin, Germany, 1993.

[31] H. X. Yi and C. C. Yang, Uniqueness Theory of Meromorphic Functions, Kluwer Academic Publishers, Dordrecht, Netherland, 2003. 